\title{
Médiévales
}

Langues, Textes, Histoire

69 | automne 2015

Travailler à Paris (XIIIe-XVIe siècle)

\section{Des paysans au Moyen Âge. Réflexions autour de trois ouvrages récents}

\section{Vincent Corriol}

\section{(2) OpenEdition}

1 Journals

\section{Édition électronique}

URL : https://journals.openedition.org/medievales/7650

DOI : $10.4000 /$ medievales. 7650

ISSN : 1777-5892

Éditeur

Presses universitaires de Vincennes

\section{Édition imprimée}

Date de publication : 30 novembre 2015

Pagination : 183-203

ISBN : 978-2-84292-444-7

ISSN : 0751-2708

\section{Référence électronique}

Vincent Corriol, «Des paysans au Moyen Âge. Réflexions autour de trois ouvrages récents », Médiévales [En ligne], 69 | automne 2015, mis en ligne le 30 novembre 2017, consulté le 23 avril 2022 URL : http://journals.openedition.org/medievales/7650; DOI : https://doi.org/10.4000/medievales. 7650 
Vincent Corriol

\section{Des paysans au Moyen Âge \\ Réflexions autour de trois ouvrages récents}

La parution récente de trois ouvrages, tous trois consacrés aux paysans du Moyen Âge, me semble révélatrice du dynamisme de la réflexion autour de l'histoire rurale médiévale. Pourtant, et paradoxalement, ces trois ouvrages ne semblent guère partager de points communs. L'ouvrage de Mathieu Arnoux traite de la croissance européenne des XII et XIII ${ }^{\mathrm{e}}$ siècles, des laboureurs, du travail, de la constitution d'un ordo laboratorum et de la construction de la société médiévale ${ }^{1}$. Celui de Nicolas Carrier est consacré au temps long de la servitude médiévale, envisagée sur un large espace alpin, de la fin de l'Antiquité aux débuts de l'ère moderne ${ }^{2}$. David Glomot, quant à lui, consacre son étude à la toute fin du Moyen Âge, sur un cadre réduit et une thématique précise, le paysage et son évolution sous l'action des sociétés humaines ${ }^{3}$.

Trois auteurs, trois périodes et trois échelles différentes, trois objets et trois méthodes différentes. Et pourtant demeure une constante qui les rapproche : la volonté de repenser les rapports sociaux au Moyen Âge, en repartant de sa base, le monde paysan. Tous trois parlent des paysans du Moyen Âge, et ce simple fait mérite d'être salué dans un paysage historiographique français où l'intérêt porté à ce groupe social se fait rare. Plane ici la figure tutélaire de Robert Fossier, qu'aucun des trois ne revendique vraiment et dans la continuité duquel ils s'inscrivent pourtant ${ }^{4}$.

1. M. ARnoux, Le Temps des laboureurs. Travail, ordre social et croissance en Europe (XI ${ }^{e}$-XIVe siècle), Paris, 2012.

2. N. CARRIER, Les Usages de la servitude. Seigneurs et paysans dans le royaume de Bourgogne ( $V I^{e}-X V^{e}$ siècle), Paris, 2012.

3. D. GLOMOT, "Héritage de serve condition ». Une société et son espace : la Haute Marche à la fin du Moyen Âge, Limoges, 2013.

4. Je pense notamment à Paysans d'Occident : XI ${ }^{e}$-XIVe siècles, Paris, 1984, à Hommes et villages d'Occident au Moyen Âge, Paris, 1992, et surtout à son Enfance de l'Europe : $X^{e}$ XII siècle. Aspects économiques et sociaux, Paris, 1982 (Nouvelle Clio, 17). 
Il est certes un peu rapide de parler sans autre forme de procès d'abandon ou de déclin de l'histoire rurale chez les médiévistes français dans les dernières décennies. La publication régulière de belles études montre que le champ conserve de beaux restes ${ }^{5}$. Et pourtant : demeure ce sentiment persistant d'un champ peu à peu déserté au profit de thèmes perçus comme plus novateurs ou peut-être plus attractifs - le politique, l'espace social, les représentations, l'espace tout court, dont l'ouvrage de David Glomot me semble représentatif : parler d'une société rurale mais, à travers son espace, le paysage et la manière dont elle influe sur ce dernier ${ }^{6}$.

\section{Servage relatif, servage absolu}

Malgré son titre ${ }^{7}$, le livre de David Glomot ne consacre qu'une part réduite au servage, confirmant finalement que, malgré un renouveau certain des études qui lui sont consacrées, rares encore sont les historiens à faire de la servitude un objet central de leurs recherches ${ }^{8}$. Un seul sous-chapitre, sur les dix-sept chapitres de l'ouvrage, lui est spécifiquement consacré (« Comment peut-on être $\operatorname{serf}^{9}$ ? »), soit trente-sept pages sur les quatre cent vingt pages de texte. Place pour le moins réduite au regard du surtitre, mais qui correspond parfaitement au projet et à l'optique de l'auteur et de son ouvrage : faire une histoire des paysages et des sociétés humaines qui les ont façonnés. Le servage ne constitue pas une problématique majeure de l'ouvrage. Que l'on ne voie pas ici une quelconque critique : l'auteur

5. On me pardonnera de ne pas en dresser ici une bibliographie un tant soit peu complète ; la tâche aurait largement excédé le cadre de ce point de vue.

6. Le terme de paysage, largement façonné par l'action humaine, est préféré par l'auteur à celui d'environnement, conçu comme davantage intemporel : « Il faut donc à présent chercher à comprendre comment l'homme agit dans l'environnement et produit des paysages, par la rencontre entre un milieu à évolution lente et des structures sociales, économiques et politiques par essence temporaires, voire éphémères » (D. GLOMOT, "Héritage de serve condition »..., p. 16).

7. D. GLOMOT, «Héritage de serve condition »...

8. Outre l'ouvrage de Nicolas Carrier déjà mentionné et sur lequel je vais revenir plus loin, on peut citer Les Formes de la servitude : esclavage et servage de la fin de l'Antiquité au monde moderne, Actes de la table ronde de Nanterre, 12 et 13 décembre 1997, Mélanges de l'École française de Rome - Moyen Âge, 112/2 (2000), p. 493-631; La Servitude dans les pays de la Méditerranée occidentale chrétienne au XII siècle et au-delà : déclinante ou renouvelée ?, Actes de la table ronde de Rome, 8 et 9 octobre 1999, ibid., p. 633-1085; M. Bourin et P. FreEdman éd., Forms of Servitude in Northern and Central Europe. Decline, Resistance and Expansion, Turnhout, 2005 (actes du colloque « Nouveaux servages de l'Europe médiane et septentrionale (XIII ${ }^{\mathrm{e}}-\mathrm{XVI} \mathrm{I}^{\mathrm{e}}$ siècle) », Göttingen, février 2003) ; V. CORRIOL, Les Serfs de Saint-Claude. Étude sur la condition servile au Moyen Âge, Rennes, 2009 ; N. CARRIER éd., Nouveaux servages et sociétés en Europe (XIII ${ }^{e}$-XIXe siècle), Rennes/ Caen, 2010.

9. D. GLOMOT, « Héritage de serve condition »..., p. 262-299. 
signale lui-même la pauvreté des sources pour traiter cette question dans l'espace qu'il étudie ${ }^{10}$. Mais la raison majeure est bien que le servage ne constitue pas l'objet principal d'étude. Il se trouve qu'il y a des serfs en Haute Marche, et l'auteur y consacre quelques pages très intéressantes. Au cœur de sa réflexion, il y a cependant l'environnement, le paysage et les interactions entre des sociétés humaines et leur milieu. L'introduction de la première partie, qui sert d'introduction générale, est sans ambiguïté à ce sujet $^{11}$ :

L'histoire, médiévale en particulier, s'inscrit dans cette réflexion contemporaine sur la gestion de l'environnement, puisque c'est au Moyen Âge que certaines des plus importantes structures de peuplement et de mise en valeur des territoires européens sont nées, ou ont acquis une configuration durable : habitats, réseaux routiers, finages, structures agraires s'esquissèrent tout particulièrement aux temps médiévaux.

Il ajoute un peu plus loin que son projet est de « chercher à comprendre comment l'homme agit dans son environnement et produit des paysages, par la rencontre entre un milieu à évolution lente et des structures sociales, économiques et politiques par essence temporaires, voire éphémères ${ }^{12} »$. Se revendiquant historien géographe ${ }^{13}$, David Glomot est finalement moins l'historien d'une société que celui d'un paysage rural dont les sociétés humaines ne seraient qu'une composante, même si l'auteur évite le piège d'un anthropocentrisme historique déterminant. Il s'inscrit finalement dans une tradition historiographique française, celle des grandes études régionales; mais une historiographie contemporaine, résolument ancrée dans le XXI ${ }^{e}$ siècle, qui, au-delà de l'étude des réseaux seigneuriaux ou des relations économiques, privilégie celle des espaces, des territoires, des paysages, des interactions de l'homme et des sociétés et de leur environnement.

Paradoxalement, la vision du servage proposée ici reste beaucoup plus traditionnelle et tributaire de schémas académiques. Certes, l'auteur entérine ce qu'il faut considérer désormais comme acquis, telle l'absence de lien entre statut juridique et statut économique, même s'il n'est pas toujours exempt d'une relative ambiguïté à ce sujet. Il fait aussi justice du lien souvent établi entre servage, d'une part, et structures familiales complexes - telles qu'associations fraternelles contractuelles ou communautés familiales élargies -, d'autre part. Beaucoup moins convaincante est sa tentative de

10. Ibid.,p. 111.

11. Ibid., p. 15.

12. Ibid., p. 16.

13. Ibid., p. 24 : «L'historien des campagnes est d'abord un géographe.» 
distinction entre un servage personnel, attaché à la personne et relevant d'un Moyen Âge central finissant, et un servage réel, qui ne toucherait que la terre et viendrait supplanter cette première forme. David Glomot y voit deux servages distincts, l'un du XIII ${ }^{\mathrm{e}}$ siècle qui disparaît, l'autre du XIV ${ }^{\mathrm{e}}$ siècle qui apparaît, sans cependant chercher à voir les éventuelles continuités qui sous-tendent cette évolution, ne serait-ce que celle du vocabulaire ${ }^{14}$. Là où l'auteur manifeste cependant de la manière la plus évidente la prégnance de pesanteurs historiographiques, c'est dans son insistance à présenter le servage comme un archaïsme et une manifestation du conservatisme des seigneuries ecclésiastiques où il se manifeste majoritairement, alors même qu'il démontre que ce servage qu'il voit s'intensifier et se répandre dans la seconde moitié du $X^{\mathrm{e}}$ siècle diffère de ses avatars antérieurs. S'il y a création de nouvelles formes serviles ou, à tout le moins, évolution et adaptation des statuts sociaux à des conditions nouvelles, peut-on alors parler d'archaïsme ? Le servage qui se dessine aux XIV et $X^{\mathrm{e}}$ siècles n'est pas le servage du XII ${ }^{\mathrm{e}}$ siècle, et cette évolution suppose une réflexion sociale qui ne saurait se résumer à une simple régression.

Au contraire de l'ouvrage de David Glomot, celui de Nicolas Carrier entend résolument s'emparer du servage médiéval ${ }^{15}$. Le projet est audacieux : aborder frontalement le servage, prendre l'objet historiographique à bras le corps sans rien éluder de sa complexité, depuis l'Antiquité jusqu'au seuil des Temps modernes, à l'intérieur d'un espace à la fois vaste et cohérent pour dépasser le simple cadre seigneurial et même celui d'une principauté, d'un royaume ou le ressort unique d'une coutume ${ }^{16}$. Ce projet est mené à partir d'une documentation et d'une bibliographie que l'auteur connaît bien, celle des Alpes, à commencer par les principautés provençale, dauphinoise et savoyarde, sans s'interdire des incursions en Suisse centrale et orientale et jusqu'au Tyrol. L'espace choisi offre ainsi à la fois la cohérence du massif montagnard et la pluralité des situations rencontrées. Le but, proclamé dès l'introduction, est ambitieux : rien moins que proposer un modèle explicatif du servage, envisagé comme un fait commun à l'ensemble des espaces et des périodes médiévales. L'auteur entend démontrer que le servage constitue

14. L'auteur est ici aux prises avec l'une des éternelles contradictions de l'historien face au servage. Il cite (p. 189) la coutume, qui fait de l'homme un serf en raison de sa terre, justifiant ainsi une présomption de servage réel. Mais la condition de l'homme est bien personnelle : si la terre fait de l'homme un serf, celui-ci le reste et transmet cette condition à ses descendants. L'auteur précise d'ailleurs (p. 275) que « l'acquisition de terre serves rend serf, l'acquisition de terre franches n'émancipe pas, tel est le principe du droit ». Ce « servage réel » semble alors très personnel !

15. N. CARRIER, Les Usages de la servitude...

16. L'auteur entend prendre « de la hauteur, afin de saisir d'un seul coup d'œil une région plus vaste ; du recul, de manière à ne pas envisager du servage que son ultime avatar » (ibid., p. 9), et souhaite « considérer la servitude médiévale dans son ensemble», en brisant «le cloisonnement entre les trois sous-périodes canoniques » (ibid., p. 10). 
un seul et même fait social unique et typiquement médiéval en perpétuelle évolution, dont les multiples variations et résurgences font qu'il ne disparaît jamais complètement du paysage social.

Ce faisant, Nicolas Carrier aborde courageusement ce qui constitue sans doute l'un des plus vieux débats de l'histoire médiévale. Qu'est-ce que le servage ? Rares sont les historiens qui oseraient s'aventurer à répondre sans précaution à cette question ! À commencer par la terminologie, précisée par Nicolas Carrier : servitude est à entendre dans un sens général (« condition juridique caractérisée par l'absence au moins formelle de liberté $\left.{ }^{17} »\right)$, servage dans le sens précis du type de servitude que connaît l'Europe occidentale chrétienne au Moyen Âge. Esclavage et servage sont deux types spécifiques de servitude.

Cette question de la terminologie concentre les difficultés d'approche et d'interprétation de la servitude depuis longtemps. Le droit médiéval a toujours été très clair, suivant en cela la formule latine Omnes homines aut liberi aut servi sunt. C'est précisément tout le problème auquel se confronte Nicolas Carrier : celui d'une dichotomie stricte affirmée par le droit d'une part, et le constat, d'autre part, d'une infinie pluralité des situations et des appellations, dans le temps et dans l'espace, incluant parfois des «nonserfs » dont tout laisse à penser qu'ils le sont, et des serfs que rien ne semble distinguer des libres ! Difficultés lexicales que les historiens ont parfois contribué à embrouiller : servitude, servage, jusqu'à la non-liberté ou aux «demi-libres »de Pierre Boutruche ${ }^{18}$ (mais si ces hommes ne sont pas libres, que sont-ils au regard du droit médiéval sinon des serfs ?).

Cette question du servage a été l'objet de beaucoup d'attention de la part de Marc Bloch et de ses successeurs, sans que rien de définitif n'ait pu entraîner une acceptation générale de la part de la communauté médiéviste. Demeure le constat, que j'ai moi-même dressé il y a quelques années, et que Nicolas Carrier dresse à nouveau : le servage médiéval, du fait de ce paradoxe initial (un statut unique, une opposition radicale entre libre et serf proclamée par le droit ; une pluralité des appellations et des conditions) nous échappe toujours. Ce qui en fait un objet fascinant, car il constitue sans doute le concept le plus éminemment médiéval des sociétés du Moyen Âge européen. Le point nodal est celui des liens d'homme à homme, en

17. Ibid., p. 10 .

18. R. Boutruche, Seigneurie et féodalité, Paris, 1970, t. II, p. 53 : « La liberté personnelle n'était pas constamment entière. Entre elle et la servitude flottait une masse confuse d'états intermédiaires [...]. Comment appeler les hommes placés dans l'entre-deux ? Demi-libres ? [...] Des scribes du XIII siècle n'emploient-ils pas le terme "semi liber" ? ». Il cite aussi les termes de « liberté restreinte » ou de « très petite liberté », eux aussi attestés au Moyen Âge. 
une proximité évidente, et pas seulement dans le lexique, de la dépendance nobiliaire et vassalique avec la dépendance servile ${ }^{19}$.

Peut-on alors, à la manière de David Glomot, continuer à voir des degrés dans le servage ? Nicolas Carrier s'y risque, établissant une typologie des redevances en fonction de leur lourdeur et de leur poids, réel ou supposé, déterminant un servage plus ou moins lourd. Il peut ainsi définir plusieurs types de servage en fonction des temps et des lieux : « se distinguent un servage personnel fort et une mainmorte faible et théorique, ainsi qu'un servage ecclésiastique et un autre laïque ${ }^{20} \gg$. Autant de types de servage toujours opposés au statut des libres, comme si celui-ci était unique.

Il y a là quelque chose de troublant : définir le servage par des charges spécifiques, puis chercher à en définir le degré, la lourdeur, semble presque logique, sans que rarement le même processus soit mis en avant pour la liberté. Inconsciemment, c'est bien toujours à un absolu, la liberté, que l'on oppose des formes de servage, comme si l'on n'arrivait jamais tout à fait à aller au bout de la conclusion : le servage, la liberté ; des formes serviles, des libertés. C'est sans doute ce qui explique pour une part la fascination qu'exerce le servage à nos yeux : il nous pousse in fine à interroger une notion essentielle à nos yeux contemporains, qui n'est pas tant celle de la dépendance que celle de la liberté.

Les médiévistes ont déjà largement répondu à cette question de la liberté : la liberté médiévale est une liberté relative, jamais absolue. Elle est à entendre au pluriel, dans le sens « libre de ». C'est une autorisation, une permission, une licence, une « franchise », jamais un concept. Rares pourtant sont les historiens à parler de degré dans la liberté, comme on parle de degré dans le servage, en fonction de la lourdeur des redevances et des contraintes qui pèseraient sur les libres. Si les médiévistes ont compris depuis longtemps qu'il fallait distinguer concept et modalités d'application, soit les libertés (modalités pratiques du statut libre) et la liberté (le statut des libres), il faut nécessairement faire de même avec le servage : des servages (modalités pratiques du statut servile), le servage (le statut des serfs). C'est précisément ce à quoi s'emploie Nicolas Carrier, et c'est à mes yeux une avancée majeure : nous contraindre à penser le servage comme un

19. Le fait avait déjà été souligné par M. BLOCH, La Société féodale, Paris, 1939-1940 (2 vol.), rééd. 1968 et 1994, p. 209 : «Être l'homme d'un autre homme : dans le vocabulaire féodal, il n'était point d'alliance de mots plus répandue que celle-là, ni d'un sens plus plein. Commune aux parlers romans et germaniques, elle servait à exprimer la dépendance personnelle, en soi. Cela, quelle que fût par ailleurs la nature juridique précise du lien et sans que l'on s'embarrassât d'aucune distinction de classe. Le comte était l'homme du roi comme le serf celui de son seigneur villageois [...]. L'équivoque ne choquait point, parce qu'en dépit de l'abîme entre les rangs, l'accent portait sur l'élément fondamental commun : la subordination d'individu à individu. »

20. N. CARRIER, Les Usages de la servitude..., p. 267. 
absolu, dont les formes peuvent varier ; de la même manière que la liberté est un absolu, dont les formes peuvent varier en fonction des temps, des lieux et des individus. C'est finalement le grand paradoxe du servage qui est en partie résolu, et ce n'est pas peu : Nicolas Carrier nous propose de penser le servage comme la liberté, c'est-à-dire à la fois comme un concept juridique (le servage, la liberté) et comme un objet relatif, dont les formes et les modalités pratiques peuvent être éminemment variables et s'avérer parfois étonnamment proches, jusqu'à nier l'opposition conceptuelle qui les caractérise.

C'est bien ce que rappelle Nicolas Carrier : mettre en évidence la relativité du regard de l'historien. Le servage est un absolu (on est serf ou libre, sans autre possibilité) et il est dans le même temps relatif : tout dépend de quel point de vue on se place ! Il faut écouter les anciens : «Quis potest esse servus unius et liber homo alterius », remarquait déjà Bracton au XIII ${ }^{\mathrm{e}}$ siècle ${ }^{21}$, nous offrant ainsi une autre grille d'interprétation. On peut donc faire du servage une lecture très différente : juridique et binaire, ou sociale et protéiforme, mouvante, floue, jusqu'à devenir imperceptible. D'où l'inutilité de chercher à identifier des signes, charges ou redevances qui marqueraient à coup sûr la servitude des serfs : toute servitude est nécessairement relative, mais toute liberté l'est tout autant : «Le servage, sous toutes les formes qu'il a prises successivement, correspond toujours à des servitudes limitées, partielles, relatives : en fait, des dépendances, mais considérées comme servitude du fait de leur ressemblance avec cette servitude absolue qui sert de référence ${ }^{22}$. » Il ne faut pas se laisser prendre à la rigueur des mots, qui disent esclavage, et aux réalités nécessairement atténuées qu'ils désignent. C'est seulement en articulant cette distinction entre un absolu, marqué par un vocabulaire esclavagiste et binaire, et un relatif, marqué par la diversité des situations des paysans tenanciers, que l'on parvient alors à envisager le servage. C'est là l' " ambiguïté essentielle au servage, qui consiste à considérer comme absolu ce qui est relatif ${ }^{23} \gg$. Le servage est finalement une façon de parler, une « phraséologie » pour Marc Bloch, d'ailleurs cité par Nicolas Carrier, qui insiste ${ }^{24}$ :

21. Henry de Bracton, De legibus et consuetudinibus Angliae ( $\left.\mathrm{f}^{\circ} 197 \mathrm{~b}\right)$, consultable sur Harvard Law School Library online (http://bracton.law.harvard.edu/Unframed/Latin/ v3/101.htm, consulté le 27 mai 2015). Cité par F. JOUON DES LONGRAIS, « Le vilainage anglais et le servage réel et personnel », dans Le Servage, Bruxelles, 1937 (Recueils de la société Jean Bodin pour l'histoire comparative des institutions, II), éd. complétée et mise à jour en 1959, rééd. Paris, 1984, p. 201-242.

22. N. CARRIER, Les Usages de la servitude..., p. 347.

23. Ibid., p. 349.

24. Ibid., p. 188. 
En ses origines, le servage est un nom, une qualification qu'on choisit de mettre sur une dépendance préexistante : cette dépendance, dorénavant, sera appelée servitude, ce qui ne la changera pas fondamentalement mais permettra de la consolider. Le servage ne découle pas de l'esclavage ; c'est l'étiquette « esclavage » qu'on met sur autre chose, comme on pourrait y mettre, ailleurs ou dans d'autres circonstances, l'étiquette « liberté ».

Et plus loin : « À certains égards en effet, il n'est qu'un nom qu'on peut apposer sur tout lien de dépendance : qu'on le mette sur une charge nouvelle, un nouveau type de contrainte, et on a un "nouveau servage" 25 ". À l'historien de faire la part de la conception profondément nominaliste des juristes du Moyen Âge, qui en parlant de servage ne désignent pas un concept désincarné, mais une réalité particulière ; et le terme sert à qualifier des réalités différentes dans leurs manifestations concrètes, mais semblables dans leur conception : "Si nous voyons apparaître des serfs dans la documentation, c'est qu'un scribe a choisi de considérer comme servitude ce qui pourrait être autrement qualifié, et nous apparaît alors comme une simple dépendance, d'origine banale ou plus souvent foncière ${ }^{26}$. »

Choisir le servage comme objet de réflexion peut sembler désuet au regard de questionnements résolument contemporains : environnement, interaction entre hommes et milieux, avec en toile de fond l'inévitable question de l'adaptation au changement climatique ; autant d'interrogations actuelles, ce que le servage n'est plus, trop lié à la dépendance, à la domination sociale, à une conception dépassée de la société où les rapports et les conflits entre groupes sociaux étaient au centre du débat public. Le problème du servage, et c'est précisément l'un des facteurs qui rendent son analyse si complexe, est peut-être qu'il ne fait pas communauté, quand notre réflexion sociale est obnubilée par le fait communautaire. Il se fond dans les communautés religieuses, politiques, professionnelles, sociales ou familiales, qu'il transcende et ignore ; il n'est le marqueur d'aucun groupe social $^{27}$. Dans la vie quotidienne, rien ou si peu ne distingue les serfs des libres, et l'historien peut très bien passer complètement à côté des serfs s'il ne les cherche pas, ou si la documentation choisie ne les mentionne pas. Cette difficulté est d'ailleurs signalée par David Glomot, confronté à des

25. Ibid., p. 348 .

26. Ibid., p. 146.

27. J'en veux pour preuve les deux volumes que le groupe « Genèse de l'anthroponymie moderne » a consacrés au servage médiéval : rien dans ce fait aussi éminemment social que l'anthroponymie ne permet de distinguer des serfs des Francs, les pratiques des deux groupes sont étonnamment semblables. Voir M. Bourin et P. Chareille éd., Genèse médiévale de l'anthroponymie moderne, t. V : Intégration et exclusion sociale : lectures anthroponymiques, vol. 1 : Serfs et dépendants au Moyen Âge (VIII'-XII siècle); vol. 2 : Serfs et dépendants au Moyen Âge (Le «nouveau servage »), Tours, 2002. 
formes de servage qui ne disent pas forcément leur nom : "Même si les expressions "homme de serve condition" ou "mortaillable" n'apparaissent que presque honteusement, il ne faut pas être dupe : les redevances exigées des tenanciers sont caractéristiques de la condition servile ${ }^{28}$. »J'ai eu l'occasion de constater moi-même que, selon les sources choisies, un même individu peut être présenté comme serf ou non, en fonction de l'économie du document et de sa finalitée ${ }^{29}$. Préciser le statut de quelqu'un dans un document a un sens; ne rien en dire ne permet de tirer aucune conclusion valable. C'est précisément l'ambiguïté du servage et l'intérêt de la réflexion à son sujet : il est une inégalité socio-juridique institutionnalisée qui ne se traduit pas forcément par une inégalité sociale ou économique et qui ne constitue à aucun moment un facteur identitaire.

\section{Travail, servage et société}

Mathieu Arnoux, dans sa passionnante étude consacrée à l'ordo laboratorum, ne s'intéresse pas directement au servage, qui ne constitue pas une catégorie spécifique des travailleurs du Moyen Âge. Le point de départ de sa réflexion est le formidable mouvement de croissance qui affecte l'Occident médiéval entre l'an mil et le milieu du XIII ${ }^{\text {e }}$ siècle. Croissance qui reste pourtant l'un des parents pauvres de la recherche médiévale : «il y a quelque chose de frustrant à rechercher dans la bibliographie récente une analyse convaincante de la croissance médiévale ${ }^{30} »$. Son hypothèse initiale est que la seule possibilité d'explication convaincante de cette croissance réside dans le travail paysan, une « révolution industrieuse ${ }^{31}$ », qui se traduit par une hausse forte et durable de l'offre de travail $^{32}$ :

Si l'on tient pour acquis qu'une croissance économique soutenue s'établit en Europe à partir de la fin du $\mathrm{X}^{\mathrm{e}}$ siècle, et se prolongea jusqu'au milieu du $\mathrm{XIII}^{\mathrm{e}}$ siècle, et que ce mouvement s'établit sans changement des conditions

28. D. GLOMOT, « Héritage de serve condition »..., p. 191.

29. Ainsi les accensements, qui peuvent préciser ou non le statut du preneur : $c f$. V. CORRIOL, Les Serfs de Saint-Claude..., p. 163 et suiv.

30. M. ARnOUX, Le Temps des laboureurs..., p. 10.

31. Mathieu Arnoux emprunte ce concept à l'économiste Jan de Vries, qui caractérisa la phase initiale du décollement économique de la première révolution industrielle par un accroissement du travail et de sa productivité, considéré par les travailleurs comme le moyen d'une plus grande indépendance. Cf. J. DE VRIES, The Industrious Revolution. Consumer Behaviour and the Household Economy, 1650 to Present, Cambridge, 2008. De manière significative, c'est une citation de cet auteur qui ouvre son ouvrage.

32. M. ARnOUX, Le Temps des laboureurs..., p. 13. L'auteur présente ainsi son projet : donner à voir "le processus de "révolution industrieuse" à l'œuvre dans les campagnes européennes avant la peste noire » (ibid., p. 342). 
techniques de la production, il convient de s'interroger sur le moteur de cette croissance. La seule hypothèse convaincante est qu'elle résulta d'une augmentation massive et durable des surfaces cultivées et des fruits récoltés. Le problème principal est d'alors d'expliquer une telle évolution, c'est-àdire de comprendre ce qui poussa les habitants des campagnes à intensifier leurs efforts.

Aucune autre hypothèse, croissance démographique ou progrès technique, ne résiste sérieusement à l'analyse. L'auteur avance alors l'idée centrale que ce mouvement de croissance économique des $\mathrm{X}^{\mathrm{e}}-\mathrm{XIII}^{\mathrm{e}}$ siècles est rendu possible

par une reconstruction de la hiérarchie sociale autour de la notion de travail, entendu comme travail des champs, allant de pair avec la construction d'un paysage agraire ordonné en terroirs communautaires et structuré sur des espaces de circulation des subsistances, à l'intérieur des communautés ou le long des itinéraires commerciaux ${ }^{33}$.

Au centre de cette nouvelle hiérarchie, la réflexion sur l'idéologie tripartite s'impose alors. Le travail est au cœur de la réflexion sociale et de la constitution d'un ordo laboratorum qui en découle, le travail devenant à la fois un des piliers de la société chrétienne et le « vecteur principal de l'identité sociale ${ }^{34} »$ du monde paysan. En s'affirmant comme critère unique de structuration de ce troisième ordre, le travail impose de transcender les divisions qui parcourent le monde paysan en une unification radicale qui regroupe presque toute la population. On touche ici aux vieux débats de la fin de l'esclavage antique et des «mutations » sociales des $\mathrm{XI}^{\mathrm{e}}$ et XII ${ }^{\mathrm{e}}$ siècles : asservissement généralisé ou émancipation complète de la paysannerie ? La focalisation du débat vers la mise en place du système seigneurial détourne l'attention du monde paysan. Or, dans l'optique de l'auteur, cette mise en place n'est pas le point principal : libre ou non, le paysan travaille, et c'est précisément là à la fois sa fonction, ce qui le définit et ce par quoi il se définit.

La revendication du travail comme facteur identitaire permet donc de transcender le vieux clivage libre/non libre, mais ne le fait pas disparaître pour autant. La distinction servile n'est alors guère valide et n'apporte rien à la réflexion. Si Mathieu Arnoux n'accorde pas d'attention particulière au servage, c'est que celui-ci ne constitue pas une catégorie spécifique 
du travail. Là encore, le servage n'apparaît pas ici comme un critère discriminant au sein de la société médiévale ${ }^{35}$ :

Un point crucial : dans le nord-ouest du continent, l'avènement d'un ordo laboratorum va de pair avec l'émancipation du travail paysan : même là où il est asservi ou enserré dans les liens de la domination seigneuriale, le laboureur n'est pas dépossédé de sa personne, mais reste le sujet de sa propre histoire $^{36}$.

L'auteur envisage ici la liberté comme la reconnaissance de l'individu en tant que sujet, opposant liberté à esclavage et faisant du servage un cas particulier de cette liberté. Position qui n'est pas très éloignée de celle de Nicolas Carrier, qui considère que le passage de l'esclavage au servage s'effectue avec la reconnaissance légale du mariage, de la possession et de l'hérédité, autre manière de faire de l'individu dépendant non un objet mais un sujet.

Mathieu Arnoux assume le caractère partiel, et peut-être même partisan de l'analyse, revendiquant la volonté délibérée d'écarter tout ce qui contrevient au schéma proposé ${ }^{37}$. Et on s'engouffrera dans la brèche qu'il nous propose : la démonstration est stimulante, passionnante, mais laisse de côté la question du travail contraint, en un mot de la corvée qui, de manière surprenante, demeure absente de son approche. Le seul moment où l'auteur aborde la question du travail contraint se situe dans l'introduction, à propos des facteurs d'explication avancés des mécanismes de la croissance médiévale. Il constate que, si le travail paysan a pu être avancé comme l'un de ces facteurs, il est cependant toujours considéré comme un acquis, qu'il s'agisse d'un travail volontaire ou d'un travail forcé, la contrainte seigneuriale et la pression fiscale devenant source et moteur de cette intensification du travail. Ce faisant, il ne distingue finalement pas travail contraint et travail librement consenti. Mais comment alors expliquer l'adhésion des paysans à la conception d'un ordre dont la valeur centrale qui les définit, le travail, leur est par ailleurs parfois imposée comme une contrainte par les autres ordres, via la corvée?

35. Pas plus que les choix anthroponymiques déjà signalés. Fabrice Mouthon dresse un constat similaire dans les communautés villageoises : « Il est tentant de penser qu'au sein du village médiéval, la première forme de distinction sociale passe par le statut juridique. Il n'en est rien pourtant, car si la société féodale hérite de la période précédente la dichotomie entre libres et non-libres, elle la relègue assez rapidement au second plan. En outre, avant la fin du XII ${ }^{\mathrm{e}}$ voire avant le XIII ${ }^{\mathrm{e}}$ siècle, la définition juridique de la liberté et du servage reste assez floue » (F. Mouthon, Les Communautés rurales en Europe au Moyen Âge, Rennes, 2014, p. 138).

36. M. ARnOUX, Le Temps des laboureurs..., p. 97.

37. Ibid., p. 348 : «L'analyse qui est présentée ici a choisi d'ignorer ou de contourner ce qui pouvait menacer sa cohérence. » 
La grille d'analyse, qui se situe résolument sur un mode économique, explique sans doute en partie cette absence paradoxale : le projet de l'auteur est bien de construire un modèle explicatif de la croissance médiévale des $\mathrm{X}^{\mathrm{e}}-\mathrm{XII}{ }^{\mathrm{e}}$ siècles, dont le travail, constitutif de l'identité du troisième ordre, serait la clé. Cependant si on peut lire la corvée à l'aune d'une approche strictement fiscale (la corvée comme une réquisition de travail, une forme spécifique d'imposition en nature), c'est oublier un peu vite sa dimension anthropologique et sociale : travail forcé, la corvée est aussi, et peutêtre même avant tout, contrainte de domination exercée sur le corps des dominés. Et c'est peut-être ce facteur qui rend finalement la mention de la corvée inutile aux yeux de l'auteur. Cette contrainte imposée contribue précisément à souligner la distinction entre l'ordo laboratorum et les autres. Le travail demeure alors bien un critère spécifique de distinction entre les ordres, non plus comme une revendication des travailleurs adhérant à un idéal commun, mais comme une contrainte imposée par la classe dominante qui ainsi se distingue des autres qui, précisément, travaillent.

Contraint ou librement consenti, le travail constitue un facteur d'adhésion interne ou d'identification externe d'un ordo défini par sa tâche. Se pose alors nécessairement la question de l'adhésion des paysans à cette mise en ordre imposée. L'auteur n'est pas dupe ${ }^{38}$ :

L'accord sur la société d'ordre reposait, sans doute au vu et au su de tous, sur une tension ou sur un malentendu : l'ordre n'est pas le même pour ceux qui travaillent et pour ceux qui consomment les fruits de ce travail. Pour les laboratores, l'ordo était revendication sociale et religieuse avant, voire plus, qu'obéissance à l'injonction providentielle énoncée par les clercs.

L'interrogation rejoint ici les doutes de Fabrice Mouthon au sujet de l'adhésion des populations paysannes à cette nouvelle idéologie tripartite ${ }^{39}$ :

$\mathrm{Au}$ vu des résistances rencontrées, comme de l'évolution ultérieure cependant, il y a fort à parier que cette intériorisation est largement surestimée par l'historiographie. Les élites elles-mêmes ne semblent en tout cas pas dupes et entretiennent une véritable psychose du soulèvement paysan.

Mais pour Mathieu Arnoux, il ne faut pas non plus sous-estimer cette adhésion qui donne à l'idéologie tripartite sa pleine puissance. La force de cette idéologie réside précisément dans le degré d'adhésion de chacun à l'ordre qui lui est proposé : il ne suffit pas de rejeter tous ceux qui ne sont

38. Ibid., p. 342.

39. F. Mouthon, Les Communautés rurales..., p. 94. 
pas oratores ou bellatores dans une même catégorie, celle des travailleurs (laboratores), pour qu'elle acquière une existence. S'il est possible de parler d'adhésion, c'est aussi parce que la théorie des trois ordres permet de dépasser l'affrontement seigneur/paysan pour proposer un projet de société : « sortir de l'affrontement, encore symbolique, entre paysans et seigneurs, pour entrer dans une voie de négociation qui requiert la reconnaissance de la dignité sociale des deux parties ${ }^{40} »$. Cette conception d'un ordre des travailleurs donne aux paysans un rôle, une place et une fonction sociale ; elle leur permet de s'intégrer à un projet social commun, avec conscience de son importance : sans travail paysan, pas d'autre ordre possible. En d'autres termes, les textes normatifs ne sont ni programmatiques ni prospectifs, mais l'enregistrement d'un état de fait et doivent composer avec des réalités existantes. Ils supposent une reconnaissance mutuelle de chacune des composantes pour sortir de l'affrontement. Le fait même que les activités laborieuses puissent obtenir une véritable reconnaissance sociale, quand les élites politiques et religieuses revendiquent pour elles-mêmes oisiveté et contemplation, incite à penser que l'ordo laboratorum lui-même contribue à cette élaboration.

\section{Système social, système économique}

Si des passerelles existent entre ces trois ouvrages, les divergences sont aussi profondes : références, périodes envisagées, ampleur chronologique, échelle spatiale contribuent à les éloigner. Et pourtant les points de convergences qui se dessinent entre eux me semblent révélateurs d'une approche éminemment contemporaine de l'histoire en France, reflétant les questionnements du corps social contemporain. Avant toute chose, on notera que les trois ouvrages témoignent d'un dépassement de la querelle mutationniste ainsi que de celle de la «naissance du village »; deux thèmes et leurs continuations qui ont dominé les années 1980 et 1990. L'approche proposée par chacun de ces trois ouvrages s'inscrit pleinement dans l'histoire économique et sociale, celle-là même revendiquée par Robert Fossier ${ }^{41}$, en une sorte de retour à des fondamentaux quelque peu délaissés, mais un retour qui prendrait en compte les évolutions de son temps. Signe en est la focalisation sur les paysans, les hommes et leur mode de vie, en les considérant comme acteurs et comme sujets, non comme une donnée invariante et passive de l'histoire des campagnes : « je reste convaincu que les paysans qui représentèrent alors l'écrasante majorité de la population

40. M. ARnOUX, Le Temps des laboureurs..., p. 128.

41. R. Fossier, L'Histoire économique et sociale du Moyen Âge occidental : questions, sources, documents commentés, Turnhout, 1999 («L'Atelier du médiéviste », 6). 
de l'Europe ne peuvent être définis adéquatement comme non-clercs, nonnobles et non-urbains, ni décrits comme de simples agents animés d'une histoire qui leur échappait. Ils sont le sujet de ce livre », conclut Mathieu Arnoux dans la dernière phrase de l'ouvrage.

Faire des paysans non plus des objets d'étude mais des sujets de l'histoire : l'objectif est partagé aujourd'hui par nombre de médiévistes qui tentent d'approcher, d'une manière ou d'une autre, le monde paysan et de rendre aux masses paysannes un rôle actif. C'est l'ambition partagée par exemple par Laure Verdon ${ }^{42}$, et par Fabrice Mouthon qui, de manière un peu provocatrice mais salutaire, ouvre l'ouvrage qu'il consacre aux communautés paysannes par cette remarque ${ }^{43}$ :

Les chimpanzés font de la politique. Les primatologues nous l'assurent [...]. Les paysans du Moyen Âge, quant à eux, ne faisaient pas de politique. Ils travaillaient, faisaient des enfants, se plaignaient, se révoltaient parfois, mais leur esprit ne s'élevait jamais jusqu'à la sphère politique. C'est du moins ce qui ressort, avec un peu de provocation, de la lecture d'une large partie de l'historiographie médiévale contemporaine. Les paysans n'auraient joué aucun rôle majeur dans l'histoire politique du Moyen Âge si ce n'est, ponctuellement, celui de victime de l'exploitation, de la répression, ou du mépris des classes dirigeantes.

En ceci ces ouvrages nous placent au cœur de l'histoire sociale : envisager le monde paysan comme une société à part entière, dans toute sa diversité. Un peu comme si l'anthropologie historique avait mis près de quarante ans à atteindre le monde paysan. C'est précisément ce que fait David Glomot lorsqu'il met en relation des structures sociales (métayers et petits paysans, serfs et libres) avec les contraintes techniques et locales, confrontant les structures d'exploitation et leur rentabilité économique. C'est ce que fait Nicolas Carrier quand il tente de percevoir les mécanismes qui régissent cet invariant perpétuellement fuyant qu'est le servage, conçu dans la perspective d'un Marc Bloch comme un fait social majeur et spécifiquement médiéval avant toute autre considération. C'est enfin ce que fait Mathieu Arnoux lorsqu'il s'intéresse aux laboureurs, érigés ici en symbole d'un ordre conçu et présenté comme pilier de la société, y compris et à commencer par ceux qui ne font pas partie de cet ordre.

42. L. VERDON, La Voix des dominés. Communautés et seigneurie en Provence au bas Moyen Âge, Rennes, 2012.

43. F. Mouthon, Les Communautés rurales..., «Introduction », p. 7. Même ambition revendiquée par Jean-Pierre Devroey pour le haut Moyen Âge : « conserver à la paysannerie son autonomie comme sujet de l'histoire » (J.-P. DEVROEY, Puissants et misérables. Système social et monde paysan dans l'Europe des Francs (VI'-IXe siècle), Louvain-la-Neuve/ Bruxelles, 2006, p. 359). 
Peut-on se borner à déplorer le silence des sources concernant les paysans, constatant qu'elles n'abordent les paysans que de l'extérieur et par en haut, par l'élite seigneuriale et politique, intellectuelle et religieuse, celle qui écrit et surtout laisse des écrits ? C'est précisément tout l'enjeu : « comment les définir et les décrire autrement qu'en évoquant les pouvoirs qui s'imposent à eux ${ }^{44}$ ? » Non sans raison, Mathieu Arnoux constate que le débat sur les évolutions sociales des $\mathrm{X}^{\mathrm{e}}-\mathrm{XII}{ }^{\mathrm{e}}$ siècles a surtout porté sur les transformations sociales, leur nature et leur rythme (la fameuse mutation), les hiérarchies et la domination, laissant de côté les aspects proprement économiques de cette évolution. La croissance qui en constitue la toile de fond est un fait acquis et finalement non discuté, éclipsé par l'importance prise par les notions de seigneurie et féodalité dans la construction de la période. Or ces changements sont à la fois économiques et sociaux; mais la focalisation du débat sur la mutation a fait passer sous silence la question économique. Les paysans et la constitution d'un troisième ordre sont souvent restés les parents pauvres de la réflexion : «l'hypothèse que [la paysannerie] puisse contribuer de manière dynamique et innovante à la construction de l'économie et de la société n'est simplement pas envisagée ${ }^{45}{ }^{2}$.

On en vient alors à l'un des aspects les plus marquants à mes yeux de ces ouvrages, qui marquent un véritable basculement : c'est précisément cet intérêt consacré à l'économie. Chacun à leur manière, ils témoignent d'un retour en force de la réflexion économique ${ }^{46}$. Curieusement, c'est le plus jeune des trois qui manifeste la vision la plus orthodoxe de l'économie. David Glomot oppose par exemple de manière convaincante des pratiques de gestion différenciées dans les seigneuries ecclésiastiques et les seigneuries laïques. L'interprétation qu'il en fait est cependant discutable : l'absence d'innovation, de renégociation ou d'augmentation substantielle de la rente foncière dans les accensements et réaccensements, qui se font toujours selon les coutumes et devoirs anciens chez les seigneurs ecclésiastiques, est interprétée, à la suite de Jean Tricard ${ }^{47}$, comme une preuve de leur absence de sens de l'innovation, leur frilosité pour la nouveauté et leur conservatisme quasi naturel, opposé à un esprit d'entreprise et d'initiative qui caractériserait nombre de seigneurs laïques. Ainsi, le choix de privilégier, lors du processus de reconstruction de la seconde moitié $\mathrm{du} \mathrm{XV}^{\mathrm{e}}$ siècle, les baux à long terme, est présenté comme désavantageux pour les seigneurs,

44. M. Arnoux, Le Temps des laboureurs..., p. 201.

45. Ibid., p. 203.

46. Cette dimension n'est cependant guère perceptible chez Nicolas Carrier, et pour cause : la question économique est volontairement laissée de côté, car elle aurait perturbé la démonstration et la réflexion, de manière à la déconnecter de celle du servage et de s'affranchir de ce lien encore trop souvent établi entre servage et pauvreté.

47. J. TRICARD, Les Campagnes limousines $d u$ XIV au XVe siècle. Originalité et limites d'une reconstruction rurale, Paris, 1996. 
dont la rente risque de s'éroder avec le temps et l'inflation. Mais on peut aussi renverser le point de vue : à long terme, ce type de baux est aussi davantage favorable aux paysans, dont l'intérêt mérite, tout autant que celui des seigneurs, d'être pris en compte !

Ces conditions de concession de la terre peu favorables aux établissements seigneuriaux ecclésiastiques, mais favorables aux paysans, ne peuvent être interprétées sans autre forme de procès comme une méconnaissance des mécanismes économiques chez les seigneurs ecclésiastiques ou comme une absence de prise de conscience d'une efficacité économique que l'auteur ne définit par ailleurs jamais. À le lire cependant, on comprend vite que cette efficacité économique est conçue comme celle $\mathrm{du} \mathrm{XXI}^{\mathrm{e}}$ siècle, fondée sur la recherche d'un profit financier tangible et substantiel, sans finalement se poser la question de la validité de ces concepts contemporains pour des sociétés anciennes. Or la question doit être posée : des critères, autres que strictement économiques, peuvent entrer en jeu, qui ne sont pas envisagés ici. Georges Duby l'avait déjà signalé dans son étude sur la seigneurie templière provençale ${ }^{48}$. La seigneurie n'est pas forcément une structure rentable au sens contemporain du terme : elle ne rapporte pas forcément plus que ce qu'elle nécessite de frais et d'investissement. Et pourtant elle peut être éminemment rationnelle en termes économiques, à condition de mettre en rapport les concepts économiques utilisés avec ceux de la société concernée.

En l'occurrence, et dans le cas précis qu'envisage David Glomot, l'absence d'innovation n'est peut-être pas seulement immobilisme ou archaïsme. Le conservatisme est aussi un gage de stabilité ; et c'est aussi le rôle d'un établissement ecclésiastique que de favoriser la stabilité des fidèles. Ce conservatisme vise précisément à un retour à l'équilibre initial, celui d'une société instaurée par Dieu dont l'ordre a été perturbé. L'innovation devient alors une nouveauté dont il convient de se méfier. Appliquer sans plus de précaution des raisonnements économiques contemporains à des sociétés anciennes régies par d'autres conceptions ne va pas sans risque et constitue même une erreur méthodologique. La raison économique, pour des religieux, est précisément de faire fructifier le don de Dieu et de conserver cette stabilité sociale pensée comme idéale ${ }^{49}$. Il ne faut pas oublier que les seigneurs ne peuvent pas toujours imposer leur volonté ; et que leur intérêt économique (à nos yeux) n'est pas forcément leur intérêt

48. G. Duby, « La seigneurie et l'économie paysanne. Alpes du sud, 1338 », Études rurales, 2 (1961), p. 5-36.

49. On lira à ce sujet avec profit et très grand intérêt les très belles pages consacrées à cette conception économique par V. TONEATTO, Les Banquiers du Seigneur : évêques et moines face à la richesse (IVe-début du IXe siècle), Rennes, 2012, p. 313 sq. 
tout court. La rationalité économique telle que nous la concevons n'est pas forcément celle de l'époque ${ }^{50}$.

De la même manière, l'auteur pose aussi la question, extrêmement pertinente, de la possibilité d'une influence du fait servile sur le paysage, en une approche à ma connaissance très novatrice. Mais cette approche est en permanence influencée par un présupposé : d'emblée, le servage est suspecté de freiner initiatives et développement ; et ce, alors même que l'auteur démontre la disparition, au cours du XV siècle, d'anciennes formes de servage et l'évolution de nouvelles. Au passage, il relève que le servage fait preuve ici, à la manière de ce que montre aussi Nicolas Carrier, d'une étonnante plasticité dans ses adaptations locales et d'une étonnante résilience : toujours moribond, toujours renaissant. Considéré comme un archaïsme au regard d'un progressisme social constamment sous-jacent dans la réflexion de l'auteur, le servage est nécessairement conçu comme un handicap au développement et à la prospérité. D'où ce titre un peu surprenant, « Comment peut-on être serf ? ».

Suivons son analyse. David Glomot part du constat que « les métayers comme les comparsonniers ${ }^{51}$ ont des façons spécifiques de travailler le sol et de produire un paysage nettement dissocié et identifiable, sous la forme de grandes exploitations aux morphologies $\operatorname{compactes}^{52} »$. Il pose alors logiquement la question de savoir si le renouveau servile entraîne lui aussi une modification des paysages, ou tout du moins, a une traduction dans l'utilisation de l'espace et la création du paysage : « le serf n'est pas un misérable, mais son rang réputé inférieur a forcément une incidence sur sa façon de travailler et d'utiliser l'espace ${ }^{53} \gg$. Il se situe ici résolument sur le terrain de la géographie contemporaine, explicitement citée ${ }^{54}$. L'auteur met en évidence quelques nuances entre patrimoine servile et patrimoine

50. Le monde médiéval n'est pas forcément un monde anté-économique et peut élaborer sa propre rationalité économique. Voir J.-P. DEVROEY, Puissants et misérables..., notamment le chap. 16, intitulé «Conclusions (pour de nouveaux chantiers) : un monde raisonnable ?» (p. 585-611), et plus particulièrement les points II ( « La rationalité et la question de la nature des sociétés pré-modernes », p. 588) et IV (« La question de la rationalité économique », p. 600) : «Chez l'historien ou l'anthropologue, l'hypothèse de l'irrationalité est un préjugé idéologique. Le thème de la rationalité économique apparaît donc à des époques et sous des formes différentes dans le souci de déterminer les règles de la bonne gestion, du bon gouvernement d'entités collectives comme la maison-famille (Aristote, Xénophon), le monastère (saint Benoît) ou l'ordre institutionnel (la res publica carolingienne) » (p. 601).

51. Membres d'une communauté familiale paysanne.

52. D. GLOMOT, « Héritage de serve condition »..., p. 262.

53. Ibid., p. 262.

54. Ibid., p. 262, n. 109 : «Cette problématique emprunte beaucoup à la géographie, plus particulièrement aux questions de front écologique dans les pays émergents, où les paysages portent les marques des inégalités socio-économiques. » Je note que l'auteur, s'il puise des références parfaitement justifiables, ne prend guère de gants pour les appliquer sans grande précaution à un tout autre contexte. Je noterai aussi que ces références concernent des 
franc : plus morcelé et dispersé dans le finage, il se distingue de l'héritage franc finalement davantage par une question de degré que de nature : « s'il ressemble beaucoup à la petite tenure franche, l'héritage de serve condition se distingue par sa fragilité : maisons modestes, parcelles ridicules, affreusement nombreuses et éparses, rareté des herbages et des étables ${ }^{55} \gg$. L'auteur ne peut cependant déterminer très clairement ce qui ressort, dans ce constat, de la pauvreté et de la servitude ; constat extrêmement difficile à faire au demeurant, la comparaison avec les exploitations des métayers ou des frérèches ne pouvant servir de contre-exemple suffisamment convaincant.

L'analyse présente cependant un premier biais, qui consiste à opposer métayers et comparsonniers, d'une part, aux serfs, de l'autre, quand il a pu démontrer par ailleurs que les structures d'exploitations ou les structures familiales n'entretenaient pas de relation avec le statut, servile ou non ${ }^{56}$. Le plus étonnant reste le présupposé initial de son approche. David Glomot part du principe que le servage réactivé est un déclassement, et donc ne peut que produire une «dégradation » du paysage : « la question majeure est de savoir si, dans l'hypothèse d'un durcissement du servage, les territoires subissent une forme de dégradation, reflet du déclassement social ${ }^{57}$ ». L'hypothèse pourrait être recevable, mais elle mériterait des clarifications : qu'est-ce qu'une « dégradation » du territoire ? Et, à l'inverse, que serait une « valorisation » du territoire ? Ce présupposé est ici problématique à double titre. D'abord parce que cette présentation laisse deviner une conception archaïque du servage comme statut infâmant et dégradant pour l'individu, ne pouvant qu'entraîner une incidence sociale forte et dévalorisante. Une macule servile qui se transmettrait au paysage, en quelque sorte : à statut dégradé, paysage dégradé. Ensuite parce que l'auteur présuppose qu'un territoire valorisé est un territoire économiquement rentable, au sens où peuvent l'entendre des géographes et des économistes du XXI ${ }^{\mathrm{e}}$ siècle. Or je suis loin d'être certain que la rentabilité économique, telle que nous la percevons aujourd'hui, soit bien la même au Moyen Âge et puisse être appliquée sans précaution à des réalités médiévales que l'on ne maîtrise encore qu'imparfaitement.

Il me semble que la principale faille réside dans cette conception économique qui fait prévaloir le développement et la rentabilité sur tout autre critère. Si une telle conception est bien celle qui régit l'économie actuelle, il est en revanche téméraire de l'appliquer sans précaution à des réalités médiévales qui savent témoigner d'autres conceptions économiques ; j'en veux pour preuve la fascinante étude de Valentina Toneatto consacrée à

inégalités « socio-économiques », quand le servage se situe davantage dans une dimension socio-juridique, qui ne recoupe pas les clivages économiques.

55. Ibid., p. 296.

56. Ibid., p. 244.

57. Ibid., p. 262. 
ce sujet ${ }^{58}$, et les travaux de Giacomo Todeschini ${ }^{59}$. C'est précisément l'un des attraits de l'ouvrage de Mathieu Arnoux, qui témoigne ici d'un intérêt renouvelé des médiévistes et, au-delà, des historiens, pour d'autres conceptions et d'autres systèmes économiques. Sans doute n'est-ce pas un hasard si des ouvrages comme ceux de Mathieu Arnoux ou de Valentina Toneatto paraissent maintenant, à un moment de crise persistante où l'interrogation sur les modèles économiques qui prévalent aujourd'hui se fait insistante. Il s'agit, pour ces historiens comme pour les économistes dit « hétérodoxes ${ }^{60}$ », de réinterroger le monde, les relations sociales, les mécanismes économiques et les liens qui unissent les deux.

Le choix des sources effectué par Mathieu Arnoux se place dans cette optique. En optant délibérément pour des sources littéraires et narratives, et non des sources de la pratique, il se détache du productivisme quantitatif qui imprègne parfois l'histoire économique pour se rapprocher d'une conception plus sociale de l'économie, certains diront moins rationnelle, plus subjective. Le propos est alors davantage celui d'une histoire de l'économie, comprise comme une science humaine et sociale, détachée d'une réflexion mathématique, que de l'histoire économique an sens strict ; position résumée avec un brin de malice par une première partie

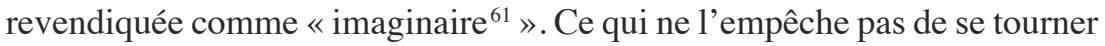
ensuite vers d'autres sources plus conventionnelles; en témoignent les

58. V. TONEatTo, Les Banquiers du Seigneur...

59. G. TODESCHINI, I mercati et il tempio. La società christiana et il circolo virtuoso della richezza fra Medio Evo ed età moderna, Bologne, 2002 ; ID., Richezza francescana. Dalla povertà volontaria alla società di mercato, Bologne, 2004 (trad. fr. : Richesse franciscaine. De la pauvreté volontaire à la société de marché, Lagrasse, 2008).

60. Sans entrer dans le détail de la querelle, le débat médiatique qui confronte des positions « orthodoxes » (à comprendre comme néo-libérales) et des positions « hétérodoxes » (qui s'y opposent) caricature une réflexion épistémologique qui remonte au début du XX⿳⺈冂大 s., à l'origine des courants dits « institutionnalistes » et « néo-classiques ». Cette discussion est en partie une opposition méthodologique (économétrie et modélisation économique $v s$ enquête socio-politique) : voir à ce sujet le blog d'O. BouBA-OLGA, professeur des universités en aménagement de l'espace et urbanisme à la Faculté de sciences économiques de l'Université de Poitiers [http://blogs.univ-poitiers.fr/o-bouba-olga/2015/05/15/guerre-entre-economisteselements-de-reflexion/], consulté le 29 mai 2015. Mais ce positionnement renvoie aussi à une épistémologie plus fondamentale : pour les institutionnalistes, dont les « hétérodoxes » se revendiquent, la réflexion économique ne peut être détachée de celle concernant le système social et politique dans lequel elle s'inscrit. Voir F. S. LEE, «Être ou ne pas être hétérodoxe : réponse argumentée aux détracteurs de l'hétérodoxie », Revue française de socio-économie, 8/2 (2011), p. 123-144.

61. Cette dimension est pleinement assumée par Mathieu Arnoux, qui entend approcher son sujet via des sources essentiellement littéraires : «On ne trouvera dans les pages qui suivent ni donnée chiffrée ni tableau statistique [...]. Les mots écrits, parce qu'ils témoignent d'une communauté - celle du scripteur et de ses lecteurs, pour commencer - peuvent constituer une voie d'accès vers le réel [...]. Le même raisonnement, qui conduit à préférer les sources écrites aux données matérielles, amène en effet à privilégier les sources narratives et littéraires par rapport aux sources archivistiques, plus familières aux historiens, et à accorder 
pages que l'auteur consacre aux marchés, aux moulins et à la dîme, objet d'une passionnante réflexion.

\section{En guise de conclusion}

La référence à Karl Polanyi et à la « grande transformation », explicitement cités par Mathieu Arnoux, me semble constituer un signe de cette inflexion de l'histoire économique ${ }^{62}$. Il semble presque incongru de rappeler que l'économie et la société de marché, telles que nous les définissons aujourd'hui, ne sont qu'une des formes possibles de l'économie et du système social ; et qu'analyser la société médiévale à l'aune de critères et de concepts élaborés des siècles plus tard demeure problématique. Et sans doute n'est-ce pas totalement un hasard si les références revendiquées, Marc Bloch ou Karl Polanyi par exemple, ont été mûries dans les années trente, vécues à la fois comme une crise économique et une crise aux racines et aux conséquences plus profondes, non sans écho actuel : remise en cause d'un système structurant, celui de l'économie de marché devenue cadre d'une société de marché (pour paraphraser Polanyi), plus ou moins bien tempérée par les tentatives de régulation des États. Face aux doutes qui s'élèvent quant à l'efficacité d'un système économique présenté comme sans alternative ${ }^{63}$, la question sociale se fait lancinante : quel modèle de société, pour demain comme pour le Moyen Âge ? En témoigne le succès, ne serait-ce que médiatique, d'un Thomas Piketty, et l'intérêt mondial suscité par ses analyses. La réflexion conduite par Mathieu Arnoux au sujet de la dîme s'inscrit pleinement dans ce contexte ; pour une large part, elle est aussi une réflexion sur l'impôt, sa répartition et son utilisation.

C'est aussi à ces interrogations que me semblent répondre ces trois ouvrages. Comment les sociétés médiévales ont-elles surmonté la crise de la fin du Moyen Âge ${ }^{64}$ ? Comment ont-elles su inventer la croissance ?

plus de valeur aux textes d'imagination et aux élaborations théoriques qu'aux récits des événements » (M. ARnOUX, Le Temps des laboureurs..., p. 10-11).

62. «Telle qu'elle apparaît à la lumière de ces textes, la dîme s'apparente à bien des points de vue aux institutions communautaires de redistribution décrites par Karl Polanyi comme caractéristiques des économies antérieures à la Grande Transformation » (ibid., p. 247). Voir K. POlANYI, La Grande Transformation. Aux origines politiques et économiques de notre temps, Paris, 1944, rééd. 1983.

63. On connaît bien l'acronyme TINA : «There Is No Alternative ».

64. M. Bourin, S. CARocci, F. Menant et L. To Figueras, « Les campagnes de la Méditerranée occidentale autour de 1300 : tensions destructrices, tensions novatrices », dans M. Bourin, F. Menant et L. To Figueras éd., Dynamiques du monde rural dans la conjoncture de 1300 : échanges, prélèvements et consommation en Méditerranée occidentale, Rome, 2014 (Collection de l'École française de Rome, 490), p. 683 : «Pour un historien médiéviste intéressé par les questions démographiques et économiques, les drames collectifs 
Comment ont-elles théorisé, vécu et articulé les inégalités sociales et juridiques ? Les points de jonction entre ces trois livres sont donc plus profonds qu'il n'y paraît. Parler du servage ou parler du paysage, c'est aussi parler du travail : imposer des redevances, y compris en travail mais pas seulement, à des paysans, c'est bien imposer leur travail ; concevoir le paysage comme le résultat d'une interaction entre des sociétés humaines et leur milieu, c'est bien encore parler du travail. C'est ici que le projet de Mathieu Arnoux prend tout son sens : à la base du système social médiéval, il y a toujours le travail paysan et l'exploitation qui en est faite. Et cette interrogation entre encore en résonnance avec l'une des questions majeures qui taraude notre société aujourd'hui, obnubilée par le chômage : partage du travail, taxation du capital/du travail, articulation de la « valeur travail » et de la valeur du travail ; ou comment articuler système social et système économique.

Vincent Corriol - Université du Maine-Le Mans - CERHIO (UMR 6258)

qui secouent actuellement notre planète évoquent ceux qui se sont joués aux derniers siècles du Moyen Âge. L'analogie peut paraître paradoxale si l'on pense à l'abîme qui sépare les structures actuelles de la production, des échanges et de la redistribution de celles d'il y a six ou sept cents ans. Et pourtant, l'analyse des différents champs où se développe aujourd'hui la crise montre bien des similitudes avec ce qui s'est passé en Europe aux XIII ${ }^{\mathrm{e}}$ et XIV ${ }^{\mathrm{e}}$ siècles, à commencer par le manque périodique de nourriture dont souffre de nos jours une partie de l'humanité, et qui n'est pas dû à l'insuffisance de la production alimentaire, mais à ses mécanismes de répartition. Les problèmes qui assaillent l'humanité depuis quelques années - ou dont la portée devient aujourd'hui évidente - ont leurs équivalents, mutatis mutandis, dans l'Europe des derniers siècles du Moyen Âge.» 